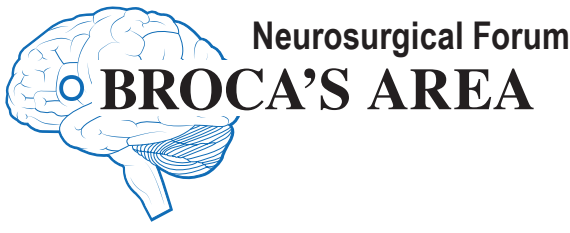

\section{Randomized controlled trials for degenerative lumbar spondylolisthesis: which patients benefit from lumbar fusion?}

\author{
Zoher Ghogawala, MD, ${ }^{1}$ Daniel K. Resnick, MD, ${ }^{2}$ \\ Steven D. Glassman, MD, ${ }^{3}$ James Dziura, PhD, ${ }^{4}$ \\ Christopher I. Shaffrey, MD, ${ }^{5}$ and \\ Praveen V. Mummaneni, MD ${ }^{6}$
}

${ }^{1}$ Alan L. and Jacqueline B. Stuart Spine Research Center, Department of Neurosurgery, Lahey Hospital and Medical Center, Burlington, MA

2Department of Neurosurgery, University of Wisconsin, Madison, WI

${ }^{3}$ Department of Orthopedic Surgery, University of Louisville School of Medicine, Louisville, KY

${ }^{4}$ Yale Center for Analytical Sciences, Yale School of Public Health, New Haven, CT

${ }^{5}$ Department of Neurosurgery, University of Virginia, Charlottesville, VA

${ }^{6}$ Department of Neurological Surgery, University of California, San

Francisco, CA

$\mathrm{P}$ ATIENTS with lumbar stenosis often suffer from symptoms of radiculopathy and claudication. If nonoperative measures, including physical therapy and epidural steroid injections, fail to control their symptoms, patients may be offered surgery. The majority of patients with lumbar stenosis do not have spondylolisthesis, and when these patients undergo surgical treatment, it is typically decompression alone, based on previously published guidelines. ${ }^{18,19}$

Some patients with lumbar stenosis also have spondylolisthesis, and this may be associated with a significant complaint of mechanical back pain. Patients with this constellation of findings may be offered lumbar decompression with or without fusion. Recently, conflicting studies have been published on the efficacy of decompression alone versus decompression with fusion for the treatment of symptomatic lumbar stenosis with spondylolisthesis. ${ }^{5,7}$

Patients with degenerative Grade I or II spondylolisthesis are not all the same. ${ }^{14}$ Some have mechanical low-back pain when there is axial load or stress on the lumbar spine, suggesting instability. Others have radiographic evidence of instability noted on dynamic plain lumbar radiographs. These nuances have been difficult to capture in prior published studies and make the generalization of study results difficult to apply to individual patients. The surgical options for treatment of lumbar stenosis with spondylolisthesis are reflected in prior lumbar guideline publications, which state that fusion should be considered in selected patients. . $^{17,20}$

The past decade has seen a focus on the efficacy of spinal decompression with or without fusion surgery, based on patient-reported outcomes. In the coming decade there will be an additional emphasis on durability, cost effectiveness, and ultimately on the value of spinal surgery. This transition from a sole focus on the efficacy of surgical treatment to an analysis of cost-effectiveness or value has become an increasingly important priority.

Our purpose is to examine the recently published randomized controlled trials that attempt to decipher the role of decompression with or without fusion for the treatment of symptomatic stenosis with spondylolisthesis and to assess the value of the addition of fusion to a decompression in the treatment paradigm.

\section{Randomized Controlled Trials}

Recently, 2 new randomized controlled trials (RCTs) evaluated the utility of adding a fusion when performing a decompressive laminectomy for lumbar stenosis with Grade I lumbar spondylolisthesis: "A randomized, controlled trial of fusion surgery for lumbar spinal stenosis" (the Swedish Spinal Stenosis Study, clinicaltrials. gov registration number NCT01994512), by Försth et al., 5 and "Laminectomy plus fusion versus laminectomy alone

ABBREVIATIONS MCID = minimum clinically important difference; ODI = Oswestry Disability Index; PCS = physical component summary; QALY = quality-adjusted life year; $\mathrm{RCT}=$ randomized controlled trial; SLIP = Spinal Laminectomy versus Instrumented Pedicle Screw.

INCLUDE WHEN CITING Published online September 23, 2016; DOI: 10.3171/2016.8.SPINE16716. 
for lumbar spondylolisthesis" (the Spinal Laminectomy versus Instrumented Pedicle Screw [SLIP] trial, clinical trials.gov registration number NCT00109213), by Ghogawala et al. ${ }^{7}$ Both studies contribute data that add to our knowledge base, although they reached different conclusions. The Swedish trial randomized 247 patients with lumbar stenosis with or without spondylolisthesis and included patients with 1 or 2 levels of disease. This trial did not include dynamic lumbar radiographs and therefore did not attempt to differentiate between those patients with radiographic instability $(>3 \mathrm{~mm}$ motion on dynamic lumbar imaging) or not. This study did not detect any difference in the treatment arms using the Oswestry Disability Index (ODI) as the primary outcome measure.

The SLIP trial, on the other hand, was a smaller study that focused upon a homogeneous population of patients with nonmobile single-level Grade I spondylolisthesis. Figures 1 and 2 illustrate the importance of defining the patient population using radiographic data. The patient in Fig. 1 has single-level spondylolisthesis with stenosis and was included in the SLIP study. The images in Fig. 2 are from a different case; the imaging demonstrates 2 levels of pathology, and this patient would therefore have been eligible for the Swedish study but would not have been eligible for the SLIP study. The SLIP investigators found superior health-related quality of life, measured using the SF-36 physical component summary (PCS) score for patients who underwent fusion in addition to decompression. This benefit was maintained at 2, 3, and 4 years following randomization. The Swedish study did not analyze the single-level spondylolisthesis cases separately, and therefore it is not possible to compare the trials directly.

Neither trial detected a difference using the diseasespecific ODI instrument, which was the primary outcome measure in the Swedish trial and a secondary outcome measure in the SLIP study. A careful examination of the data from the SLIP study suggests that single-level spondylolisthesis might be associated with better ODI scores over time. At 4 years, the difference in ODI score between groups was 9 points favoring fusion $(\mathrm{p}=0.05)$. Using a minimum clinically important difference (MCID) of 10 points for ODI, $61 \%$ of patients treated with laminectomy alone achieved the MCID for ODI, while $85 \%$ of patients treated with fusion and laminectomy achieved the MCID $(p=0.04)$. The sample-size estimate for the SLIP study was based on a published pilot study that examined the comparative effectiveness of adding lumbar fusion for patients with Grade I spondylolisthesis. ${ }^{6}$ Nevertheless, it is not clear that either trial was appropriately powered to detect a difference of 10 points even if it existed. In Tables 1 and 2, we summarize the estimates for the sample size that would be required in a future trial to detect a difference between mean ODI scores (90\% power) or to detect differences in the proportion of a patient population that achieves an MCID of 10 points for ODI. Assuming a standard deviation of 16, a total of at least 110 patients with single-level Grade I spondylolisthesis would be required to have $90 \%$ power to detect a between-groups difference of 10. These sample-size estimates do not take into account loss to follow-up. Future investigators would likely inflate these numbers by $10 \%-15 \%$ to account for interim analyses and expected rates of loss to follow-up. This lack of power is a major concern for focusing upon ODI when analyzing the results of both trials.

The SLIP study focused upon the health-related quality of life of patients with spinal stenosis and spondylolisthesis and identified a clinically meaningful improvement in those patients treated with lumbar fusion. The emphasis on health-related quality of life is consistent with a shift to patient-centered care, as seen in the orthopedic joint replacement literature. ${ }^{21}$ In addition, a focus on generic health-related quality of life is necessary for the comparison of the impact of different health interventions in our society. The magnitude of treatment benefit associated with lumbar fusion in the SLIP study is comparable to the level of improvement in health-related quality of life following hip arthroplasty. ${ }^{15}$

\section{Evidence-Based Practice Guidelines}

When creating clinical practice guidelines, a clinical question is posed. Then, the available literature is searched, graded, and interpreted to create a clinically useful recommendation to address the clinical question. In the latest version of the "Guidelines for the performance of lumbar fusion," a Grade B-level recommendation was provided, indicating moderate support for the performance of fusion in conjunction with decompression in patients with neurogenic claudication or radiculopathy due to stenosis as-
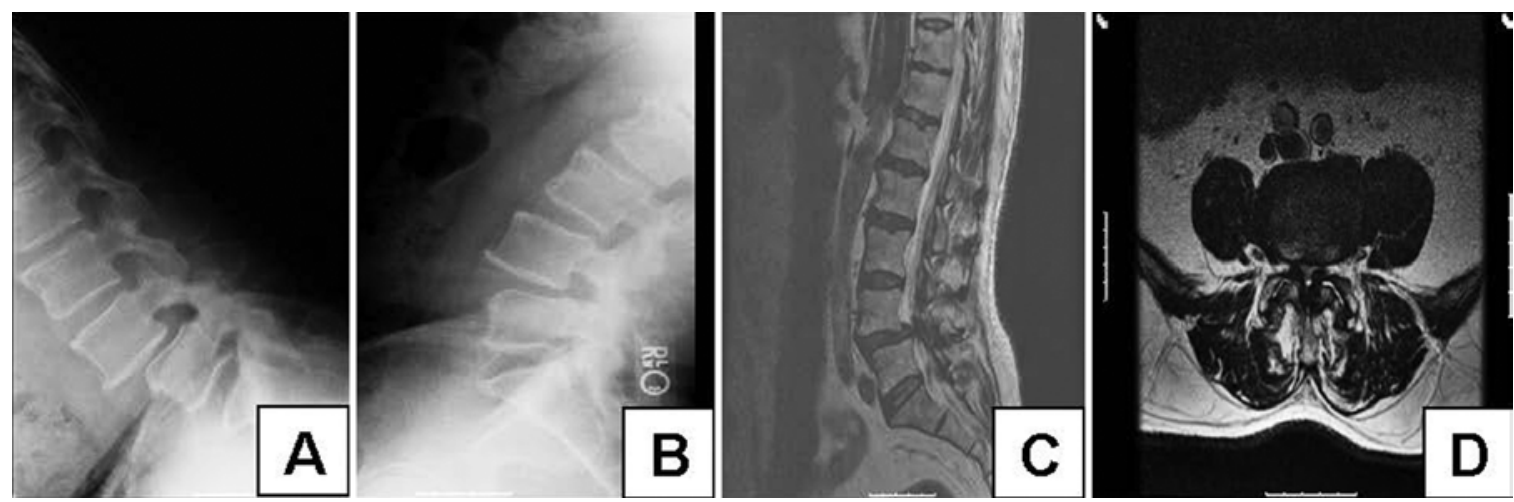

FIG. 1. Lumbar flexion (A) and extension (B) radiographs, and sagittal (C) and axial T2-weighted (D) MR images obtained in a 76-year-old man with neurogenic claudication and L4-5 spondylolisthesis. 

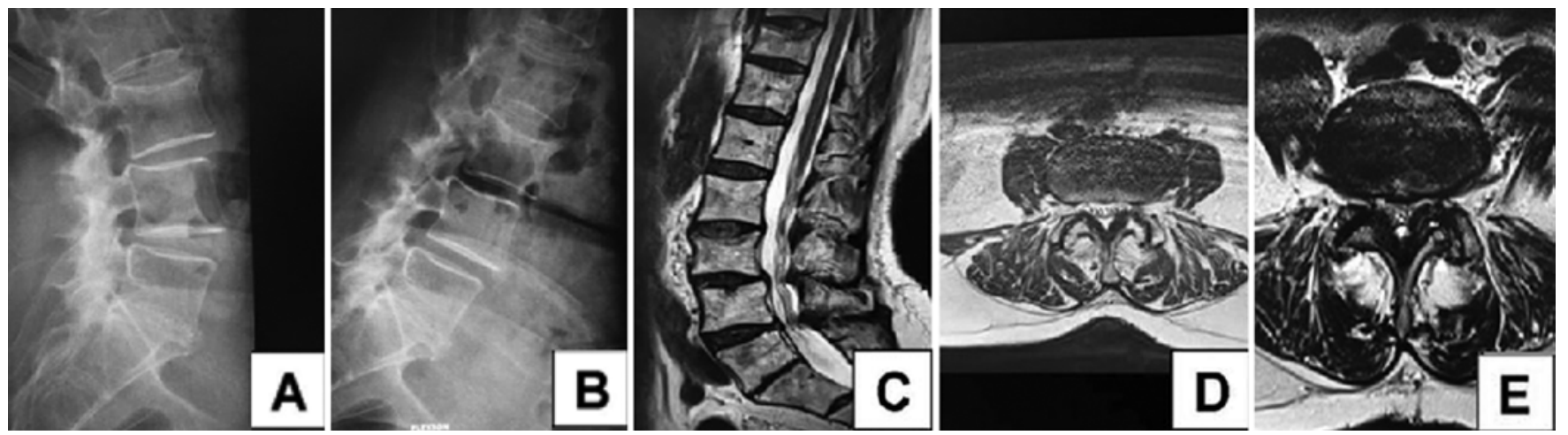

FIG. 2. Lumbar extension (A) and flexion (B) radiographs, sagittal T2-weighted MR image (C), and axial T2-weighted MR images obtained at the L3-4 (D) and L4-5 (E) levels in a 75-year-old woman with neurogenic claudication, L3-4 and L4-5 spondylolisthesis, and stenosis at the L4-5 level.

sociated with spondylolisthesis. ${ }^{20}$ The highest-quality evidence available at that time consisted of the Spine Patient Outcomes Research Trial (SPORT), which provided Level II evidence as a high-quality cohort study indicating that patients treated with laminectomy and fusion did significantly better than patients treated nonoperatively. While providing what is likely the best possible evidence for the utility of operative intervention versus contemporary nonoperative care, the study did not specifically address the issue of decompression alone versus decompression plus fusion.

Older literature addressing this particular issue exists. For example, Herkowitz and Kurz alternately assigned patients to either decompression alone or decompression followed by noninstrumented fusion..$^{10}$ In that study, patients treated with fusion reported a $96 \%$ satisfaction rate as opposed to a $44 \%$ satisfaction rate in the group treated with decompression alone. Similar results were reported in a number of other comparative trials examining decompression and decompression and fusion..$^{1,3,4,11-13,16} \mathrm{In}$ general, these studies were felt to provide relatively lowquality evidence due to the use of nonvalidated outcome measures. It has also been noted that there are multiple reports of good outcomes with decompression alone using different techniques. ${ }^{17}$ Therefore, high-quality evidence addressing the issue of whether fusion should be added to decompression in patients with spondylolisthesis was lacking until recently.

Two randomized studies dealing with this issue were published in April 2016 and are outlined above. It is appropriate to question how this new evidence could alter existing guideline recommendations. The next few paragraphs will outline the assessment of the level of evidence provided with reference to the following question: Does the addition of fusion as an adjunct to decompression in patients with claudication due to stenosis associated with spondylolisthesis improve clinical outcomes compared with decompression alone?

The first study, authored by Försth et al., ${ }^{5}$ is a randomized controlled study looking at decompression versus decompression plus fusion in the lumbar stenosis population. Outcomes were assessed via mailed surveys performed using the National Swedish Register for Spine Surgery (Swespine) at 6 months and 1,2, and 5 years. The primary outcome measure was the ODI. The authors performed a power analysis and concluded that 40 patients per treatment arm would be adequate to detect a 12-point mean difference in the ODI. ${ }^{2}$ A difference of 12 points between groups is a relatively high bar from a research standpoint. SPORT, for example, compared surgical versus nonsurgical treatment for lumbar spondylolisthesis, and the authors used a 10-point difference in ODI scores in their samplesize calculations. ${ }^{23}$ When considering MCID, it is important to consider the proportion of patients meeting this criterion. The sample-size estimates outlined in Tables 1 and 2 summarize the number of patients that would be needed in a future study using ODI. Regardless of whether a future study aims to compare mean scores or compare proportions of a population that achieve the MCID, the sample-size estimates are higher than the number of patients included in the Swedish study. Only 91 patients with single-level lumbar spondylolisthesis were included in that study (Dr. Försth, personal communication). Therefore, there is a concern that the study was underpowered to detect such a change in the ODI, particularly for patients with single-level spondylolisthesis. The authors report their results as indicating no substantial benefit for the addition of fusion to decompression.

The trial randomized 247 patients with 1- or 2-level lumbar stenosis with or without spondylisthesis into 2 surgical arms: laminectomy alone and laminectomy with fusion. It is immediately apparent that the overall population addressed in the study is not representative of the patient population most spinal surgeons are interested in learning about. The inclusion of patients without spondylolisthesis is troubling from a practical and ethical perspective given contemporary recommendations against fusion in patients with normal alignment. ${ }^{18,19}$ Therefore, the results of

TABLE 1. Sample size estimates using mean difference in ODI*

\begin{tabular}{ccc}
\hline Sample Size Estimate & Mean Difference & SD \\
\hline 172 & -8 & 16.0 \\
\hline 136 & -9 & 16.0 \\
110 & -10 & 16.0 \\
\hline
\end{tabular}

* Sample size estimates for $90 \%$ power and $5 \%$ Type I error. Sample size calculations were performed using PASS V2012. 
TABLE 2. Sample size estimates using proportion of patients achieving MCID of 10 in ODI*

\begin{tabular}{cc}
\hline Sample Size Estimate & Proportion of Patients Achieving MCID of 10 \\
\hline 144 & $60 \%$ vs $85 \%$ \\
\hline 214 & $65 \%$ vs $85 \%$ \\
\hline 348 & $70 \%$ vs $85 \%$ \\
\hline
\end{tabular}

* Sample size estimates for $90 \%$ power and $5 \%$ Type I error. Sample size

calculations were performed using Stata version 12.1 (StataCorp LP).

the primary analysis (fusion vs nonfusion irrespective of spondylolisthesis) are irrelevant to the current discussion. Ignoring the patients who did not have spondylolisthesis, the authors did analyze the results from patients with spondylolisthesis separately and found no difference in ODI, the primary outcome. This analysis appears to have been planned in the study design, so while it is a subgroup analysis, it is not post hoc and can still potentially provide high-quality evidence.

When examining the study design provided in the manuscript and in the supplemental appendices, several important issues are apparent. First, the patient population is poorly described-patients with neurogenic claudication were enrolled without characterization of the nature of spondylolisthesis and were allowed to have 1or 2-level stenosis (with or without spondylolisthesis). Patients were identified as having spondylolisthesis if there was slippage of $3 \mathrm{~mm}$ or more at a stenotic level. Patient characteristics were not further described. Back pain was neither a requirement for enrollment nor a criterion for exclusion. Dynamic instability, disc space height, facet pathology, and degree of slip were not quantified. Forty percent of patients underwent 2-level procedures; it is not clear whether spondylolisthesis was required to exist at one or both of the levels fused. The patient population is therefore clearly heterogeneous. Second, the selection of the surgical procedures was not standardized. While the majority of patients in the nonfusion group underwent a standard laminectomy with an undercutting medial facetectomy, $22 \%$ underwent a midline-sparing laminotomy. Although most patients in the fusion group underwent an instrumented posterolateral fusion using locally harvested autograft, approximately $10 \%$ were treated with either interbody or noninstrumented fusions. Additionally, the numbers of patients enrolled in each group drop off precipitously at the 5-year follow-up mark-only $138(56 \%)$ of 247 patients were available to provide data beyond 2 years. Finally, cost data were not collected beyond the 2-year time point.

Results are presented for the 2-year time point and generally do not show any significant differences between the fusion and nonfusion cohorts at 2 years. Approximately $20 \%$ of patients in both groups underwent reoperation within the study time frame. Patients who underwent 1and 2-level procedures were not separated. A post hoc analysis looking at degree of slip greater than or equal to $7.4 \mathrm{~mm}$ apparently did not show any differences between groups either, although the numbers of patients were quite small given the small cohort size to begin with.

This paper does not provide useful information for an- swering the clinical question of whether the addition of fusion adds benefit to decompression for patients with either 1- or 2-level spondylolisthesis because the patient population was heterogeneous in terms of radiographic diagnoses and number of levels treated. The paper does provide Level II evidence that the addition of a variety of fusion techniques does not have significant benefit in the first 2 years following operation compared with a variety of decompression techniques in a heterogeneous population of patients with stenosis associated with spondylolisthesis.

Ghogawala et al. published the results of the SLIP study, ${ }^{7}$ which focused on a very specific population of patients. These authors focused exclusively on patients with single-level "stable" spondylolisthesis. This patient group was chosen because there is acknowledged uncertainty as to the utility of fusion in these patients in North America. Importantly, a patient only underwent randomization if a panel of experts agreed that he or she was a study candidate. Patients were randomized to one of 2 standardized procedures - a laminectomy with medial facetectomy with or without an instrumented posterolateral fusion using iliac crest autograft. The primary outcome measure in this study was the SF-36 PCS. A power analysis was performed a priori, and both clinical and economic outcomes were followed for 4 years. Primary outcome assessment was planned for 2 years after surgery, and both cohorts had a follow-up rate greater than $80 \%$ for the primary outcome measure (SF-36 PCS) at that time point. Patients treated with fusion demonstrated modest but significant improvements in the SF-36 PCS at every time point beyond 6 weeks. Improvements were also seen in the ODI that approached significance at 2 and 4 years. Importantly, the rate of reoperation was significantly higher in the decompression-alone group than in the group treated with decompression plus fusion.

The SLIP study has some minor limitations. The main critique is that the study is relatively small and 4-year follow-up is limited to $68 \%$ of the patients. The sample-size estimate was 64 patients (based on previously published data) and was completed a priori and before any analysis was performed. Despite the study size, significant differences were found for the primary outcome. The study was underpowered to detect a difference in ODI, although a 9 -point difference was observed at 4 years $(p=0.05)$. In addition to excluding patients with radiographic instability, SLIP surgeons also excluded patients with mechanical low-back pain with presumed clinical instability. While this introduced a subjective element to the selection of patients for the study, it also represented an attempt by SLIP study surgeons to study a more homogeneous population. It should be noted that the population chosen for the study is the spondylolisthesis population felt to be least likely to require fusion in North America. The degree of facet removal was not reported in the SLIP study and may have varied among surgeons; this information might have been of interest, particularly for the laminectomy-alone group, but the intersurgeon variability represents real-world spinal surgery practice. Finally, reoperation criteria were not standardized as part of the initial study design; however, the $34 \%$ of patients in the laminectomy group who underwent reoperation had improved SF-36 and ODI outcomes 
afterward, suggesting that the reoperations in the laminectomy cohort were appropriate and effective.

In terms of level of evidence, the SLIP study provides Level I evidence for the efficacy of fusion with respect to improving clinical outcomes and achieving lower reoperation rates compared with the outcomes of standard laminectomy and medial facetectomy over a 4-year time frame in patients with neurogenic claudication associated with stable single-level spondylolisthesis. The study does not provide evidence regarding the utility of fusion in patients who have multilevel disease and does not offer evidence regarding the utility of newer minimally invasive decompression and fusion techniques.

In considering the evidence provided by both the SLIP study and the Swedish study, it is important to recognize that the patient populations treated, surgical techniques used, and outcomes measures assessed differed. Therefore, it is no surprise that results would appear to conflict on superficial review. The principle of applying a standard surgical approach to all patients with spondylolisthesis is not supported by either study. Furthermore, benefits of fusion are not expected to be realized in 1 or 2 years, and the authors of both studies are strongly encouraged to follow their respective patient cohorts as long as possible to be able to detect differences in late reoperation rates or deterioration in function due to instability or adjacent-segment degeneration. Given the state of the literature at present, either fusion or nonfusion techniques may be appropriate, depending on the patients' anatomy, lifestyle, and desires. With the advent of minimally invasive techniques for both decompression and fusion, this issue will require ongoing study.

\section{The Value of Lumbar Fusion for Degenerative Spondylolisthesis}

Health care economic value is primarily driven by 3 key factors: cost, clinical benefit, and durability. In its simplest form, "value" is expressed as cost/quality-adjusted life years (QALYs). ${ }^{9}$ While clinical benefit for spine surgery is most often reported as ODI improvement, QALYs are ideal for economic evaluation because they are preference weighted, adding a dimension of relative value to the measured improvement. QALYs are derived from health-utility measures, most commonly EQ-5D or SF-36, and health utilities have become core data elements in spine surgery research. RCTs, such as the recent studies by Försth et al. and Ghogawala et al., are an ideal setting for cost-effectiveness analysis because elimination of treatment selection bias removes one of the most significant hurdles to a meaningful assessment of "value."

Defining cost is more complex. Direct costs (instrumentation, operating room time, length of hospital stay, etc.) are most readily measured but depend somewhat on the perspective of the payer. At least in the US health care system, the hospital, private insurers, and Medicare might all consider the cost of a given hospital admission very differently. More difficult to define and collect are indirect costs (lost wages, replacement costs for homemakers, etc.), and these costs are frequently modeled rather than reported directly. One of the strengths of the Swedish study is that direct costs are not altered by payer perspective in a single-payer system, and indirect costs were specifically collected over the first 2 years of the study. ${ }^{5}$

Durability is readily defined as maintenance of clinical benefit without the need for additional interventions, but it is dependent on complete and reliable long-term follow-up that is often difficult to obtain, even in high-quality studies. Prior studies have demonstrated that interventions that are initially costly, such as lumbar fusion, may ultimately be cost-effective if they provide durable clinical benefit. ${ }^{8,22}$ The issue of durability represents the most substantial discrepancy between the Swedish study and the SLIP study and is likely to determine the actual cost-effectiveness of fusion for lumbar spondylolisthesis and spinal stenosis. Ghogawala et al. report a substantially higher revision rate in patients treated without fusion, whereas Försth et al. report equivalent revision rates. ${ }^{5,7}$ Försth et al. suggest that the higher revision rate observed in the SLIP study reflects the surgeon's belief that a subsequent procedure, fusion, might address residual symptoms after decompression alone. While this explanation might be accurate in some instances, there are multiple alternative explanations, including cultural bias toward revision surgery or a more supportive social safety net for patients unable to resume normal function in Sweden. Regardless of the rationale, if revision surgery is less common following decompression and fusion in a US population, then this treatment pathway may be more cost-effective than decompression alone, despite the higher initial cost associated with lumbar fusion.

\section{Lessons Learned and Opportunities}

Randomized controlled trials (RCTs) often produce high-quality data relative to the specific study question, but may also generate many new questions particularly when similar studies generate conflicting results. As with the recent trials reported by Försth et al. and Ghogawala et al., differences in study populations make direct comparison of the results difficult, despite the fact that both trials examine the role of lumbar fusion in patients with spinal stenosis and spondylolisthesis. In the case of the Swedish study, the heterogeneity of the study population limits the usefulness of the results. Furthermore, the lack of detail regarding imaging characteristics (disc height, facet angle, motion on dynamic imaging) makes it difficult to compare data in the Swedish and SLIP trials. An analysis of the 2 studies does not definitively answer the question of which patient requires fusion but certainly underlines the need for a better definition of clinically relevant instability in these patients. Future studies comparing the effectiveness of different surgical strategies should define patient populations as specifically as possible and perhaps include more extensive stored radiographic data for future analysis.

One of the challenges when interpreting randomized clinical studies, particularly for patients with spinal disorders, is that it is often difficult to know whether the results of these trials are generalizable to individual patients. The SLIP study found that health-related quality of life is superior for patients treated with fusion. There are clinical examples that might challenge the overall conclusions of both trials. For example, how does the SLIP study's data help with clinical decision making for an 83-year- 
old woman with severe osteoporosis, an extensive smoking history, chronic obstructive pulmonary disease, and no mechanical back pain? On the other hand, how do the Swedish study's data apply to a 58-year-old healthy man with mechanical back pain, gross motion on dynamic plain radiographs with single-level Grade I spondylolisthesis with preserved disc height? Additional studies that incorporate both radiographic and clinical variables to document complication rates following laminectomy alone or laminectomy and fusion will be necessary to supplement the findings of these RCTs.

One of the missed opportunities for both trials was that comprehensive resource utilization data were not collected as a component of long-term follow-up. As is typical for surgical trials, outpatient cost and lost productivity data were not collected prospectively. Although it might be possible to retrospectively merge claims data with the outcomes data from these RCTs to understand differences in health resource utilization, it would have been preferable to generate health-cost diaries, or some form of contemporaneous data record. Another major concept is that future studies that are funded and designed to collect long-term follow-up should not only detect reoperations but also measure direct inpatient and outpatient costs along with costs associated with lost productivity. Both of the RCTs discussed above suggest that 5 years would be the minimum length of follow-up required before conclusions could be drawn about cost-effectiveness and durability of treatment.

\section{Conclusions}

A critical analysis of the 2 recently published lumbar stenosis trials reveals several important points. The SLIP and Swedish RCTs came to different conclusions regarding the utility of lumbar fusion based on 2 important differences: patient population and outcome measure used. The Swedish study included a heterogeneous population that included patients with 1- or 2-level stenosis with or without spondylolisthesis, and radiographic details regarding instability were not captured. The SLIP study randomized a homogeneous population of patients with single-level Grade I spondylolisthesis without gross instability on flexion-extension radiographs. The Swedish study used ODI as a primary outcome measure, although the study was not adequately powered to detect a difference in treatment effect for the patients with single-level spondylolisthesis. The SLIP study was appropriately powered for the use of SF-36, a generic health-related qualityof-life instrument, and found that patients treated with laminectomy plus fusion had superior outcomes at 2, 3, and 4 years. To advance the field, RCTs will need to define patient populations and interventions specifically, to permit clinicians to effectively apply the study results to their individual patients. Finally, collecting health-resource utilization data prospectively over longer time horizons will be mandatory to document the comparative value of lumbar spinal fusion or other adjunctive interventions when performing spinal surgery.

\section{References}

1. Bridwell KH, Sedgewick TA, O’Brien MF, Lenke LG, Baldus $\mathrm{C}$ : The role of fusion and instrumentation in the treatment of degenerative spondylolisthesis with spinal stenosis. J Spinal Disord 6:461-472, 1993

2. Copay AG, Glassman SD, Subach BR, Berven S, Schuler TC, Carreon LY: Minimum clinically important difference in lumbar spine surgery patients: a choice of methods using the Oswestry Disability Index, Medical Outcomes Study Questionnaire Short Form 36, and pain scales. Spine J 8:968-974, 2008

3. Feffer HL, Wiesel SW, Cuckler JM, Rothman RH: Degenerative spondylolisthesis. To fuse or not to fuse. Spine (Phila Pa 1976) 10:287-289, 1985

4. Fischgrund JS, Mackay M, Herkowitz HN, Brower R, Montgomery DM, Kurz LT: 1997 Volvo Award winner in clinical studies. Degenerative lumbar spondylolisthesis with spinal stenosis: a prospective, randomized study comparing decompressive laminectomy and arthrodesis with and without spinal instrumentation. Spine (Phila Pa 1976) 22:2807-2812, 1997

5. Försth P, Ólafsson G, Carlsson T, Frost A, Borgström F, Fritzell P, et al: A randomized, controlled trial of fusion surgery for lumbar spinal stenosis. N Engl J Med 374:1413-1423, 2016

6. Ghogawala Z, Benzel EC, Amin-Hanjani S, Barker FG II, Harrington JF, Magge SN, et al: Prospective outcomes evaluation after decompression with or without instrumented fusion for lumbar stenosis and degenerative Grade I spondylolisthesis. J Neurosurg Spine 1:267-272, 2004

7. Ghogawala Z, Dziura J, Butler WE, Dai F, Terrin N, Magge $\mathrm{SN}$, et al: Laminectomy plus fusion versus laminectomy alone for lumbar spondylolisthesis. N Engl J Med 374:14241434,2016

8. Glassman SD, Polly DW, Dimar JR, Carreon LY: The cost effectiveness of single-level instrumented posterolateral lumbar fusion at 5 years after surgery. Spine (Phila Pa 1976) 37:769-774, 2012

9. Gold MR, Siegel JE, Russell LB, Weinstein MC: CostEffectiveness in Health and Medicine. New York: Oxford University Press, 1996

10. Herkowitz HN, Kurz LT: Degenerative lumbar spondylolisthesis with spinal stenosis. A prospective study comparing decompression with decompression and intertransverse process arthrodesis. J Bone Joint Surg Am 73:802-808, 1991

11. Katz JN, Lipson SJ, Lew RA, Grobler LJ, Weinstein JN, Brick GW, et al: Lumbar laminectomy alone or with instrumented or noninstrumented arthrodesis in degenerative lumbar spinal stenosis. Patient selection, costs, and surgical outcomes. Spine (Phila Pa 1976) 22:1123-1131, 1997

12. Kornblum MB, Fischgrund JS, Herkowitz HN, Abraham DA, Berkower DL, Ditkoff JS: Degenerative lumbar spondylolisthesis with spinal stenosis: a prospective long-term study comparing fusion and pseudarthrosis. Spine (Phila Pa 1976) 29:726-734, 2004

13. Lombardi JS, Wiltse LL, Reynolds J, Widell EH, Spencer C III: Treatment of degenerative spondylolisthesis. Spine (Phila Pa 1976) 10:821-827, 1985

14. Meyerding H: Spondylolisthesis: surgical treatment and results. Surg Gynecol Obstet 54:371-377, 1932

15. Mokhtar SA, McCombe PF, Williamson OD, Morgan MK, White GJ, Sears WR: Health-related quality of life: a comparison of outcomes after lumbar fusion for degenerative spondylolisthesis with large joint replacement surgery and population norms. Spine J 10:306-312, 2010

16. Postacchini F, Cinotti G, Perugia D: Degenerative lumbar spondylolisthesis. II. Surgical treatment. Ital J Orthop Traumatol 17:467-477, 1991

17. Resnick DK, Choudhri TF, Dailey AT, Groff MW, Khoo L, Matz PG, et al: Guidelines for the performance of fusion procedures for degenerative disease of the lumbar spine. Part 9: fusion in patients with stenosis and spondylolisthesis. J Neurosurg Spine 2:679-685, 2005 
18. Resnick DK, Choudhri TF, Dailey AT, Groff MW, Khoo L, Matz PG, et al: Guidelines for the performance of fusion procedures for degenerative disease of the lumbar spine. Part 10: fusion following decompression in patients with stenosis without spondylolisthesis. J Neurosurg Spine 2:686-691, 2005

19. Resnick DK, Watters WC III, Mummaneni PV, Dailey AT, Choudhri TF, Eck JC, et al: Guideline update for the performance of fusion procedures for degenerative disease of the lumbar spine. Part 10: lumbar fusion for stenosis without spondylolisthesis. J Neurosurg Spine 21:62-66, 2014

20. Resnick DK, Watters WC III, Sharan A, Mummaneni PV, Dailey AT, Wang JC, et al: Guideline update for the performance of fusion procedures for degenerative disease of the lumbar spine. Part 9: lumbar fusion for stenosis with spondylolisthesis. J Neurosurg Spine 21:54-61, 2014

21. Rolfson O, Wissig S, van Maasakkers L, Stowell C, Ackerman I, Ayers D, et al: Defining an international standard set of outcome measures for patients with hip or knee osteoarthritis: consensus of the International Consortium for Health Outcomes Measurement Hip and Knee Osteoarthritis Working Group. Arthritis Care Res (Hoboken) [epub ahead of print], 2016

22. Tosteson AN, Lurie JD, Tosteson TD, Skinner JS, Herkowitz $\mathrm{H}$, Albert T, et al: Surgical treatment of spinal stenosis with and without degenerative spondylolisthesis: cost-effectiveness after 2 years. Ann Intern Med 149:845-853, 2008
23. Weinstein JN, Lurie JD, Tosteson TD, Hanscom B, Tosteson AN, Blood EA, et al: Surgical versus nonsurgical treatment for lumbar degenerative spondylolisthesis. N Engl J Med 356:2257-2270, 2007

\section{Disclosures}

Dr. Ghogawala reports that he was the lead author of the SLIP study, which was published in the New England Journal of Medicine. Dr. Dziura reports that he was the lead biostatistician for the SLIP study. Dr. Glassman reports an employee relationship with Norton Healthcare; a consultant relationship with, patent holder relationship with, and receipt of royalties from Medtronic; use of a research database that was funded by NuVasive; and being past president of the Scoliosis Research Society. Dr. Shaffrey reports consultant relationships with Zimmer Biomet, Medtronic, NuVasive, K2M, and Stryker; direct stock ownership in NuVasive; patent holder relationships with and receipt of royalties from Zimmer Biomet, Medtronic, NuVasive; and support of non-studyrelated efforts from DePuy-Synthes through the ISSG Foundation. Dr. Mummaneni reports a consultant relationship with DePuy Spine; direct stock ownership in Spinicity/ISD; receipt of honoraria from AOSpine; and receipt of royalties from DePuy Spine, Thieme Medical Publishers, Springer Publishing, Taylor \& Francis Group. 